'Departamento de

Patología, Instituto

Nacional de Cancerología,

México, D.F.

${ }^{2}$ Departamento de

Hematología, Instituto

Nacional de Cancerología, México, D.F.

${ }^{3}$ Facultad de Medicina,

Universidad Nacional

Autónoma de México.

Patológica, Nuevo Hospital

"Dr. Juan I. Menchaca",

México.

Recibido el 15 de junio de

2012, aceptado el $10 \mathrm{de}$ octubre de 2012.

Correspondencia a:

Dr. Alejandro Avilés Salas

Departamento de Patología Instituto Nacional de Cancerología.

Av. San Fernando 22, Sección XVI

Tlalpan, CP 14080 ,

México, D.F.

Tel: (52) 5556280400

Fax: (52) 5556280421

E-mail: alejandroaviles2001

@yahoo.com
aResidente de Anatomía

Civil de Guadalajara

\section{Hemangioendotelioma epitelioide y fusiforme de ganglio linfático. Caso clínico}

\author{
ALEJANDRO AVILÉS-SALAS 1,3, JOSÉ CRUZ TORRES-LUCATERO ${ }^{1}$, \\ XIMENA FERNÁNDEZ-SOTOa ${ }^{a}$, MYRNA CANDELARIA-HERNÁNDEZ ${ }^{2}$
}

\section{Spindle and epithelioid haemangioendothelioma of the lymph node. Report of one case}

\begin{abstract}
Primary vascular tumors of lymph nodes are extremely rare with the exception of AIDS-related Kaposi's sarcoma. The diagnosis of epithelioid haemangioendothelioma $(E H)$ is difficult to make without ancillary studies, since it is devoid of morphological features indicating its vascular nature and it may be overlooked when it appears as a primary tumor of lymph nodes. Spindle and epithelioid haemangioendothelioma $(S E H)$ is considered to be a variant of EH, which has been reported to occur exclusively in lymph nodes and the spleen. We report a 70-year-old male with chronic lymphocytic leukemia (CLL) and left cervical lymphadenopathy. An excisional biopsy was performed, and microscopically the lymph node showed effacement of nodal architecture by a tumor composed of spindle cells disposed in intersecting fascicles, and characterized by abundant eosinophilic cytoplasm, elongated nuclei and conspicuous nucleoli. A second population of cells had an epithelioid appearance with intracytoplasmic vacuoles containing red blood cells. Immunohistochemically, the tumor cells were positive for CD31 and CD34. The final diagnosis was SEH of the lymph node.

(Rev Med Chile 2013; 141: 260-263).
\end{abstract}

Key words: Haemangioendothelioma, epithelioid; Lymph nodes; Vascular neoplasms.

linfático como hallazgo incidental en un hombre de 70 años de edad con LLC.

proliferación vascular en diversas condiciones, tales como hiperplasia paracortical, enfermedad de Castleman de tipo hialino vascular, enfermedad de Kimura y linfomas de células $\mathrm{T}^{1}$. Otro tipo de proliferación vascular menos común ocurre en la transformación vascular de los senos, el pseudotumor inflamatorio y la angiomatosis bacilar $^{2}$. Por otro lado, los tumores vasculares primarios de los ganglios linfáticos diferentes del sarcoma de Kaposi son extremadamente raros $^{3}$. El hemangioma epiteloide y fusiforme (HEF) es una variante rara del hemangioendotelioma epiteloide (HE), la cual ha sido informada casi de manera exclusiva en los ganglios linfáticos y el bazo ${ }^{4}$. Informamos un caso de HEF de ganglio

\section{Caso clínico}

Paciente masculino de 70 años de edad con diabetes mellitus tipo 2 de cinco años de evolución, que inició su padecimiento 6 meses previos a su hospitalización con cuadros intermitentes de infección de vías respiratorias. Se realizaron estudios de laboratorio que mostraron: leucocitos 38,3 miles $/ \mathrm{mm}^{3}$, hemoglobina $14,7 \mathrm{~g} / \mathrm{dl}$ y plaquetas 153 miles $/ \mathrm{mm}^{3}$, motivo por el cual fue referido al Instituto Nacional de Cancerología para su evaluación y manejo. A su ingreso se encontró en buenas 


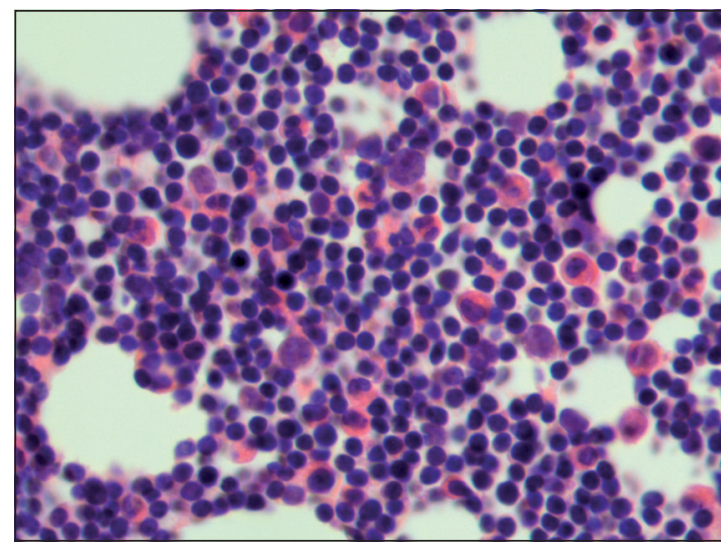

Figura 1. Biopsia de médula ósea hipercelular con sustitución de la hematopoyesis por linfocitos pequeños de contornos regulares, cromatina finamente granular, nucléolo poco aparente y escaso citoplasma (Hematoxilina-eosina, 400X).

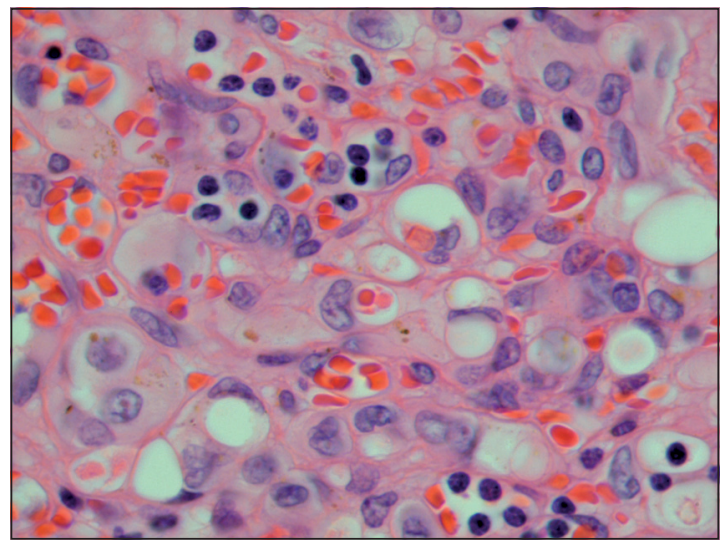

Figura $\mathbf{2 b}$. Las células neoplásicas muestran atipia nuclear moderada y citoplasma eosinófilo con ocasionales vacuolas, algunas de las cuales contienen eritrocitos (Hematoxilinaeosina, 400X).

condiciones generales y en la exploración física se palpó adenomegalia cervical izquierda de $2 \mathrm{~cm}$, de eje mayor. Se realizó nueva biometría hemática que reportó leucocitos 48,8 miles $/ \mathrm{mm}^{3}$ con $98 \%$ de linfocitos, hemoglobina de $14,9 \mathrm{~g} / \mathrm{dl}$ y plaquetas 264 miles $/ \mathrm{mm}^{3}$. Se tomó biopsia de hueso que mostró médula ósea hipercelular con sustitución de la hematopoyesis por linfocitos pequeños de contornos regulares, cromatina finamente granular, nucléolo poco aparente y escaso citoplasma (Figura 1); las células neoplásicas resultaron positivas para CD20, CD5, CD23 y Bcl-2 lo cual estableció el diagnóstico de LLC. El aspirado de médula ósea

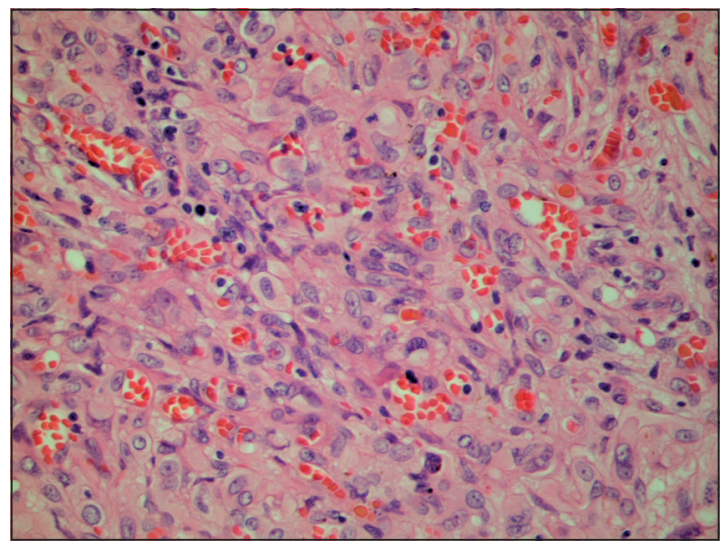

Figura 2a. Ganglio linfático con pérdida de la arquitectura por células redondas y fusiformes, dispuestas en cordones y bandas (Hematoxilina-eosina, 200X).

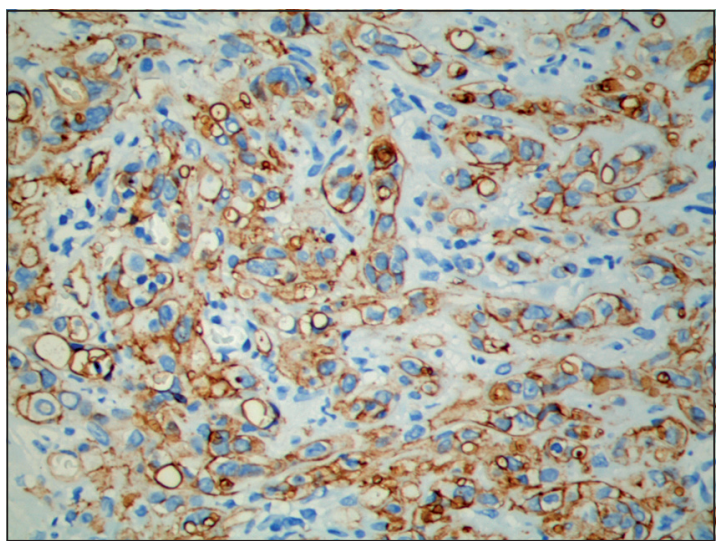

Figura 3. Expresión de CD34 en la membrana de las células neoplásicas (Técnica de inmunohistoquímica, 400X).

reportó leucemia linfocítica crónica (LLC) con el siguiente inmunofenotipo: HLA-DR 85\%, CD5 $84 \%$, CD20 74\%, CD22 77\% y CD79a 65\%. Se realizó tomografía axial computada que informó adenopatías infra y supradiafragmáticas. Posteriormente, se realizó biopsia de ganglio linfático cervical que midió 2,0 x 1,5 x 1,2 cm. En el estudio histopatológico se observó pérdida de su arquitectura a expensas de neoplasia de crecimiento sólido, constituida por células fusiformes y epitelioides dispuestas en cordones, trabéculas y fascículos cortos, inmersas en un estroma focalmente hialinizado (Figura 2a). Algunas de estas células tenían 
vacuolas citoplásmicas con ocasionales eritrocitos (Figura 2b). La atipia citológica fue moderada con escasas figuras de mitosis. Se realizaron reacciones de inmunohistoquímica utilizando anticuerpos contra: vimentina (V9; 1:150 BioGenex), CD31 (1A10; 1:50 BioSB), CD34 (QBEnd/10; 1:75 Biocare), actina de músculo liso (ASM/H12; 1:400 BioSB), CK AE1/AE3 (C11; 1:50 BioGenex), EMA (E29; 1:50 Dako), HMB45 (HMB45; 1:50 Biocare) y proteína S-100 (15E2E2; 1:5.000 BioGenex). Las células neoplásicas expresaron vimentina, CD34 y CD31 (Figura 3), que corroboró el diagnóstico de HEF de ganglio linfático.

Actualmente, el paciente se encuentra vivo, no se documentó ninguna lesión de tejidos blandos y recibe tratamiento con clorambucil y prednisona para la LLC.

\section{Discusión}

El término HE fue descrito inicialmente por Weiss y Enzinger en 1982 para un grupo de tumores vasculares de tejidos blandos con características clínicas e histológicas intermedias entre un hemangioma y un angiosarcoma, con capacidad de recurrencia local, pero raras metástasis ${ }^{5}$. Estos tumores se han informado en otras localizaciones que incluyen hueso ${ }^{6}$, pulmón ${ }^{7}$, hígado ${ }^{8}$, ovario ${ }^{9}$, mediastino ${ }^{10}$, tiroides ${ }^{11}$ y próstata ${ }^{12}$.

Los tumores vasculares primarios de ganglios linfáticos con excepción del sarcoma de Kaposi asociado a SIDA son extremadamente raros. Chan y cols. informaron una serie de 39 casos y describieron cinco grupos principales que incluían: hemangiomas, hamartoma angiomatoso, tumores vasculares epitelioides, hamangioendotelioma polimorfo y linfangiomas ${ }^{3}$.Morfológicamente, el HE está constituido por mantos y cordones de células poligonales inmersos en un estroma mixoide o hialino. Las células neoplásicas poseen un núcleo redondo o discretamente hendido, con pleomorfismo moderado y abundante citoplasma eosinófilo, que les confiere un aspecto epitelioide. En número variable las células neoplásicas tienen vacuolas citoplasmáticas que pueden contener eritrocitos, un aspecto importante de la naturaleza vascular de este tumor. Por otro lado, el HEF es considerado una variante del HE en el cual está presente un componente de células fusiformes dispuestas en fascículos cortos. Es un tumor ex- tremadamente raro que afecta casi exclusivamente los ganglios linfáticos ${ }^{3,4,13,14}$ y el bazo ${ }^{15}$. Ultraestructuralmente, las células neoplásicas muestran características de células endoteliales, incluyendo lámina basal rodeando las células neoplásicas, vesículas pinocíticas, luces intracitoplásmicas y ocasionales cuerpos de Weibel-Palade.

A diferencia del HE de tejidos blandos el cual ha sido clasificado como un tumor vascular limítrofe en base a su comportamiento clínico, la naturaleza del HEF de ganglio linfáticono ha sido determinada. A pesar de los pocos casos informados, aquellos que contaban con seguimiento estaban vivos y sin evidencia de enfermedad con una media de seguimiento de 6 años ${ }^{4,13,14}$. En base a lo anterior, se ha sugerido que esta lesión pudiera representar una proliferación reactiva de células mesenquimales inmaduras con capacidad de formación vascular, secundaria a estimulación antigénica prolongada ${ }^{4}$. En el caso informado, el diagnóstico se realizó de manera incidental, y a pesar del seguimiento corto, el paciente se encuentra vivo y se está manejando con clorambucil y prednisona para su enfermedad de base.

El diagnóstico de HE es difícil de establecer, dado que este diagnóstico habitualmente no se considera cuando se presenta como tumor primario de ganglio linfático. La naturaleza vascular del HE puede no ser aparente en el estudio histopatológico convencional y es posible confundirla con un carcinoma metastásico. El problema es por demás complejo por la inmunoreactividad ocasional de los tumores vasculares epitelioides para citoqueratina. Cuando el tumor se localiza en otros sitios se debe incluir en el diagnóstico diferencial lesiones condroides tanto benignas como malignas y lesiones fibrohistiocíticas ${ }^{6,11}$.

En conclusión el HEF de ganglio linfático es una lesión vascular rara cuyo diagnóstico puede requerir estudios auxiliares de diagnóstico tales como inmunohistoquímica y microscopia electrónica. No obstante, se debe tener en cuenta este diagnóstico en aquel tumor compuesto de grupos o cordones de células vacuoladas inmersas en una matriz mixoide-hialina.

\section{Referencias}

1. Hui PK, Chan JK, Ng CS, Kung IT, Gwi E. Lymphadenopathy of Kimura's disease. Am J Surg Pathol 1989; 13 (3): $177-86$. 
2. Chan JK, Warnke RA, Dorfman R. Vascular transformation of sinuses in lymph nodes. A study of its morphological spectrum and distinction from Kaposi's sarcoma. Am J Surg Pathol 1991; 15 (8): 732-43.

3. Chan JK, Frizzera G, Fletcher C, Rosai J. Primary vascular tumors of lymph nodes other than Kaposi's sarcoma. Analysis of 39 cases and delineation of two new entities. Am J Surg Pathol 1992; 16 (4): 335-50.

4. Cho NH, Yang WI, Lee WJ. Spindle and epithelioid haemangioendothelioma of the inguinal lymph nodes. Histopathology 1997; 30 (6): 595-8.

5. Weiss SW, Enzinger FM. Epithelioid hemangioendothelioma: a vascular tumor often mistaken for a carcinoma. Cancer 1982; 50 (5): 970-81.

6. Gómez-Arellano LI, Ferrari-Carballo T, Domínguez-Malagón HR. Multicentric epithelioid hemangioendothelioma of bone. Report of a case with radiologic-pathologic correlation. Ann Diagn Pathol 2012; 16 (1): 43-7.

7. Hristova EN, Krishnamurthy S, Ro JY, Ayala AG. Pulmonary epithelioid hemangioendothelioma with prominent signet ring cell features mimicking metastatic adenocarcinoma. Ann Diagn Pathol 2003; 7 (3): 160-4.

8. Da Ines D, Petitcolin V, Joubert-Zakeyh J, Demeocq F, Garcier JM. Epithelioid hemangioendothelioma of the liver with metastatic coeliac lymph nodes in an 11-yearold boy. Pediatr Radiol 2010; 40 (7): 1293-6.

9. Illueca C, Machado I, García A, Covisa A, Morales J,
Cruz J, et al. Uncommon vascular tumor of the ovary. Primary ovarian epithelioid hemangioendothelioma or vascular sarcomatous transformation in ovarian germ cell tumor?. Arch Gynecol Obstet 2011; 284 (6): 158991.

10. Suster S, Moran CA, Koss MN. Epithelioid hemangioendothelioma of the anterior mediastinum. Clinicopathologic, immunohistochemical and ultrastructural analysis of 12 cases. Am J Surg Pathol 1994; 18 (9): 871-81.

11. Siddiqui MT, Evans HL, Ro JY, Ayala AG. Epithelioid haemangioendothelioma of the thyroid gland: a case report and review of literature. Histopathology 1998; 32 (5): 473-6.

12. Iyer A, Thompson L, Cooper K. Epithelioid haemangioendothelioma, mimicking prostatic adenocarcinoma. Histopathology 2012; 60 (3): 522-4.

13. Silva EG, Phillips MJ, Langer B, Ordonez NG. Spindle and histiocytoid (epithelioid) hemangioendothelioma. Primary in lymph node. Am J Clin Pathol 1986; 85 (6): 731-5.

14. Napaki S, Stirling JW. Spindle and epithelioid (histiocytoid) haemangioendothelioma of cervical lymph nodes. Pathology 2004; 36 (6): 587-9.

15. Suster S. Epithelioid and spindle-cell hemangioendothelioma of the spleen. Report of a distinctive splenic vascular neoplasm of childhood. Am J Surg Pathol 1992; 16 (8): 785-92. 